\title{
Online Dynamic Smooth Path Planning for an Articulated Vehicle
}

\author{
Thaker Nayl, George Nikolakopoulos and Thomas Gustafsson \\ Control Engineering Group Department of Computer Science, Electrical and Space Engineering, \\ Luleå University of Technology, Luleå, Sweden \\ \{thanay,geonik,tgu\}@ltu.se
}

Keywords: $\quad$ Articulated Vehicle, Path Planning, Obstacle Avoidance.

Abstract: This article proposes a novel online dynamic smooth path planning scheme based on a bug like modified path planning algorithm for an articulated vehicle under limited and sensory reconstructed surrounding static environment. In the general case, collision avoidance techniques can be performed by altering the articulated steering angle to drive the front and rear parts of the articulated vehicle away from the obstacles. In the presented approach factors such as the real dynamics of the articulated vehicle, the initial and the goal configuration (displacement and orientation), minimum and total travel distance between the current and the goal points, and the geometry of the operational space are taken under consideration to calculate the update on the future way points for the articulated vehicle. In the sequel the produced path planning is being online and iteratively smoothen by the utilization of Bezier lines before producing the necessary rate of change for the vehicle's articulated angle. The efficiency of the proposed scheme is being evaluated by multiple simulation studies.

\section{INTRODUCTION}

Recently, there have been significant advances in designing automated articulated vehicles mainly for their utilization in the mining industry, where the aim has been the overall increase of the production, while making the working conditions for the human operators safer (Scheding et al., 1999). In most of the cases, these vehicles are remotely operated, while there is a continuous trend for increasing the autonomy levels, especially in the area of path planning and obstacle avoidance as the vehicles need: a) to perceive the changing environment, based on the onboard sensory systems and b) autonomously plan their route towards the final objective (Roberts et al., 2000).

For the classical task of path planning, with an obstacle detection and avoidance capability, the simplest technique to solve the problem is the altering of the vehicle's orientation, while predicting a non collision path, based on the vehicle's kinematic model, the sensing range and the safety range. In this approach a finite optimal sequence of control inputs, according to the initial vehicle position and the desired goal point is being generated, which is able to take under consideration positioning and measuring uncertainties, such that the collision with any obstacle at a given future time never occurs.

From another point of view, path planning can be divided in two main categories according to the assumptions of: a) global approaches where it is being assumed that the map is a priori available, and b) a partially known and reconstructed surrounding environment based on reactive approaches, which utilizes sensors like infrared, ultrasonic and local cameras. Characteristic examples of the first case are the RoadMap algorithm (Nilsson, 1969), the Cell Decomposition (Chazelle, 1987), the Voronoi diagrams (Guechi et al., 2008), the Occupancy Grinds (Usher, 2006) and the new Potential Fields techniques (Ge and Cui, 2000), while in most of the cases, a final step of smoothing the produced path curvatures, by the utilization of Bezier curves is being utilized (Škrjanc and Klančar, 2010).

For the second case of a partially known and online reconstructed environment, the Bug family algorithms are well known mobile vehicle navigation methods for local path planning based on a minimum set of sensors and with a decreased complexity for online implementation ( $\mathrm{Ng}$ and Bräunl, 2007). One of the most commonly utilized path planning algorithm in this category is the Bug1 and Bug2 (Lumelsky and Stepanov, 1986). Bug1 algorithm exhibits two behaviors; motion to goal with boundary following and a corresponding hit point and leave point, while Bug2 algorithm presents similar behaviors like the Bug1 algorithm, except from the fact that it tries to follow 
the fixed line from a start point to the goal, during obstacle avoidance. Other Bug algorithms that also incorporate range sensors are TangentBug (Kamon et al., 1998), DistBug (Kamon and Rivlin, 1997) and VisBug (Lumelsky and Skewis, 1990). Tangent Bug algorithm is an improvement of the Bug2 algorithm since it is able to determine the shorter path to the goal using a range sensor with a $360^{\circ}$ infinite orientation resolution. DistBug has a guaranteed convergence and will find a path if one exists, while it requires the perception of its own position, the goal position and the range sensory data (Buniyamin et al., 2011). The VisBug algorithm, needs global information to update the value of the minimum distance to the goal point, during the boundary following and for determining the completion of a loop during the convergence to the goal. In all the presented path planning algorithms, the vehicle is being modeled as a point within the world space, without any constraint in the movements, while the actual kinematics of the vehicle, which is important especially in the case of non-holonomic vehicles are being neglected.

The novelty of this article stems from the proposal of a new bug like path planning algorithm based on the dynamic model of an articulated vehicle, which is able to consider: a) the physical constraints of the vehicle, b) proper obstacle detection and avoidance, and c) smooth path generation based on an online Bezier lines processing of the produced way points. In the presented approach the solution to the path planning problem is generated online, based on partial and online sensory information of the vehicle's surrounding environment, while the path is being calculated by solving the inverse kinematic problem of the articulated vehicle or by calculating the optimal articulation angle. Moreover, as in the case of all the exploration and final goal seeking algorithms, it is assumed that the vehicle is constantly aware of the final goal coordinates. During the convergence to this goal and based on the limited range sensing of the surrounding environment, the vehicle is able to detect and avoid obstacles, while continue converging to the optimum goal. This approach is providing an online and sub optimal solution, when compared with the global path planning techniques, and it can be directly applied to the case of articulated vehicles. As it has been applied in the previous path planning algorithms for the case of a priori known space configuration, in the proposed scheme, the Bezier curves are being also utilized for filtering the produced way points and thus guarantee for an online smooth path planning due to the Bezier's line property of continuous higher-order derivatives.

The rest of the article is organized as it follows. In Section 2 the model of the articulated vehicle and the corresponding state space equations will be presented. In Section 3 the proposed novel scheme for smooth path planning and obstacle avoidance based on the articulated vehicle's dynamics will be introduced, while in Section 4 multiple simulation results will be depicted that prove the efficacy of the path planning scheme in different test cases. Finally, the concluding remarks are provided in Section 5.

\section{ARTICULATED VEHICLE MODEL}

An articulated vehicle is constructed by two parts, a front and a rear, linked with a rigid free joint, while each body has a single axle and the wheels are all non-steerable, with the steering action to be performed on the joint, by changing the corresponding articulated angle $\gamma$ between the front and the rear of the vehicle (Nayl et al., 2011) as it being also presented in Figure 1.

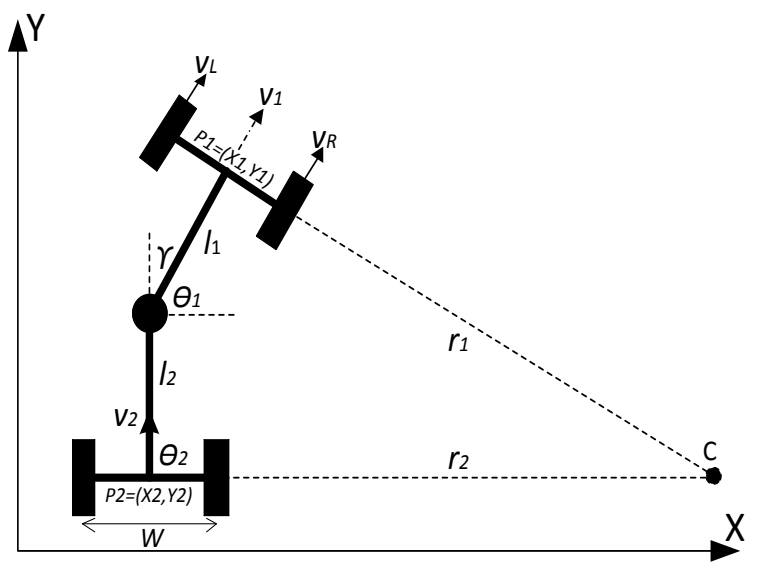

Figure 1: Articulated vehicle's geometry.

The main assumptions to derive the kinematic model of the articulated vehicle are: a) the steering angle $\gamma$ remains constant under small displacement, b) dynamical effects due to low speed, like tire characteristic, friction, load and breaking force are being neglected, c) it's assumed that the vehicle moves on a plane without slipping effects, during low-level control, the vehicle's velocities are bounded within the maximum allowed velocities, which prevents the vehicle from slipping, and c) each axle is composed of two wheels and when replaced by a unique wheel, can get:

$$
\begin{aligned}
\dot{X}_{1} & =V_{1} \cos \theta_{1} \\
\dot{Y}_{1} & =V_{1} \sin \theta_{1}
\end{aligned}
$$


The steering angle $\gamma$ is being defined as the difference between the orientation angles of the front $\theta_{1}$ and the rear parts $\theta_{2}$ of the vehicle.

The velocity $V_{1}$ at the front and $V_{2}$ at the rear parts have the same changing with respect to the velocity at the rigid free joint of the vehicle, and it can be defined by the relative velocity vector equations as it follows:

$$
\begin{aligned}
V_{1} & =V_{2} \cos \gamma+\dot{\theta}_{2} l_{2} \sin \gamma \\
V_{2} \sin \gamma & =\dot{\theta}_{1} l_{1}+\dot{\theta}_{2} l_{2} \cos \gamma
\end{aligned}
$$

where $\dot{\theta}_{1}, \dot{\theta}_{2}$ and $l_{1}, l_{2}$ are the angular velocities and the lengths of the front and rear parts of the vehicle respectively. By combining these equations it yields:

$$
\dot{\theta}_{1}=\frac{V_{1} \sin \gamma+l_{2} \dot{\gamma}}{l_{1} \cos \gamma+l_{2}}
$$

while the angles $\gamma$ and $\theta_{1}$ can be measured with a great accuracy. For the case that there is a steering limitation for driving the rear part, according to the coordinates of the point $P_{2}=\left(X_{2}, Y_{2}\right)$, the geometrical relationship between $P_{1}$ and $P_{2}$ is provided by:

$$
\begin{aligned}
X_{2} & =X_{1}-l_{1} \cos \theta_{1}-l_{2} \cos \theta_{2} \\
Y_{2} & =Y_{1}-l_{1} \sin \theta_{1}-l_{2} \sin \theta_{2}
\end{aligned}
$$

The realistic dynamic motion behavior of the articulated vehicle with initial parameters $\left[\begin{array}{llll}X_{r} & Y_{r} & \theta_{r} & \gamma_{r}\end{array}\right]$, is depicted in Figure 2, where the vehicle is requested to reach the goal destination with a specific orientation. As it can be observed, when the dynamics of the vehicle are being incorporated the motion and the overall behavior of the vehicle significantly deviates from the case where the vehicle is being considered of having the dynamics of an unconstraint point and this is one of the major contributions of this article.

The state parameters of the articulated vehicle are; $\mathbf{X}=\left[\begin{array}{lll}X & Y & \theta\end{array}\right]^{T}$ and the manipulated variables are $\mathbf{u}=$ $\left[\begin{array}{ll}V & \dot{\gamma}\end{array}\right]^{T}$, while the kinematic model of the articulated vehicle, in a state space formulation can be written as it follows:

$$
\left[\begin{array}{c}
\dot{X} \\
\dot{Y} \\
\dot{\theta} \\
\dot{\gamma}
\end{array}\right]=\left[\begin{array}{cc}
\cos \theta & 0 \\
\sin \theta & 0 \\
\frac{l_{2}}{l_{1} \cos \gamma+l_{2}} & \frac{l_{2}}{l_{1} \cos \gamma+l_{2}} \\
0 & 1
\end{array}\right]\left[\begin{array}{c}
V \\
\dot{\theta}_{\gamma}
\end{array}\right]
$$

where $\dot{\theta}_{\gamma}$ is the rate of change for the articulated angle.

\section{ON LINE SMOOTH PATH PLANNING FOR AN ARTICULATED VEHICLE}

The introduced path planning algorithm can be applied for the objective of moving a vehicle from a

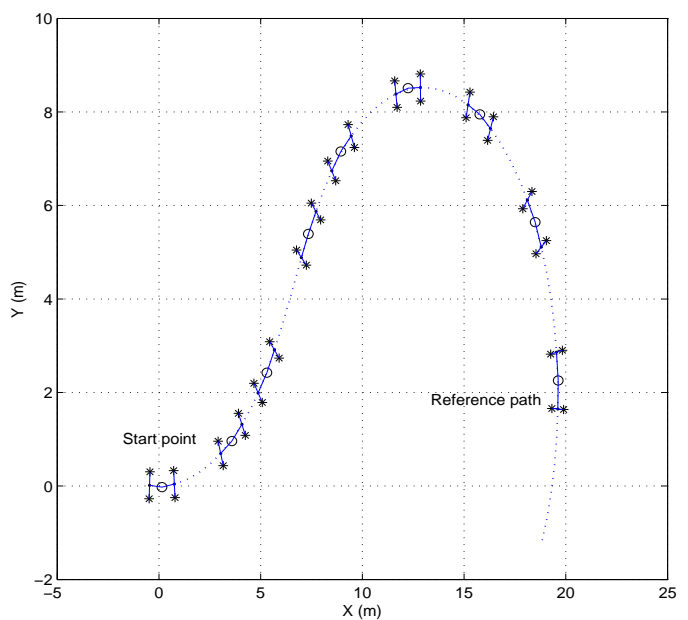

Figure 2: Realistic dynamic motion behavior of the articulated vehicle starting at $\left[\begin{array}{llll}0 & 0 & 0 & -10^{0}\end{array}\right]$ with $V=1 \mathrm{~m} / \mathrm{s}$ and $\dot{\gamma}=0^{0}$ for the first $5 \mathrm{sec}$ of movement, while $\dot{\gamma}=3.5^{0}$ for the next 6 sec to reach the goal point at [ $\left.\begin{array}{ll}20 & 2\end{array}\right]$. The vehicle dimensions are $l_{1}=l_{2}=0.6 \mathrm{~m}$ and $W=0.58 \mathrm{~m}$.

starting point to the goal point, while detecting and avoiding identified obstacles based on the real vehicles dynamic equations of motion. As a common property of the Bug like algorithms, the proposed scheme initially faces the vehicle towards the goal point, which it is being assumed to be a priori known. In the proposed path planning module it is also being assumed that the vehicle is able to online sense the surrounding environment based on the available sensory systems. The proposed scheme is able to replan the produced path, by generating new way points, after the identification of an obstacle and produce proper path deformations that need to be done for avoiding it. In all these cases the produced set of new way points are utilized as control points for a Bezier curve algorithm for online smoothing of the suggested path. The overall proposed concept of the novel path planning algorithm is being presented in the following Figure 3.

As it can be observed from this diagram the algorithm starts by defining the current position and orientation of the vehicle, denoted by $\left[X_{r}, Y_{r}, \theta_{r}\right]$ and the final goal position denoted by $\left[X_{g}, Y_{g}, \theta_{g}\right]$. Based on the onboard sensory system, the vehicle identifies the surrounding environment and obstacles and generates the way points for reaching the goal destination. In the sequel the way points are been smoothen by the utilization of Bezier filtering, while as a last step and based on the vehicles dynamics, an open loop control signal (articulated angle) is being generated to guide the vehicle. In the presented approach it is also assumed that the system is fully observable and good and timely available measurements can be provided 


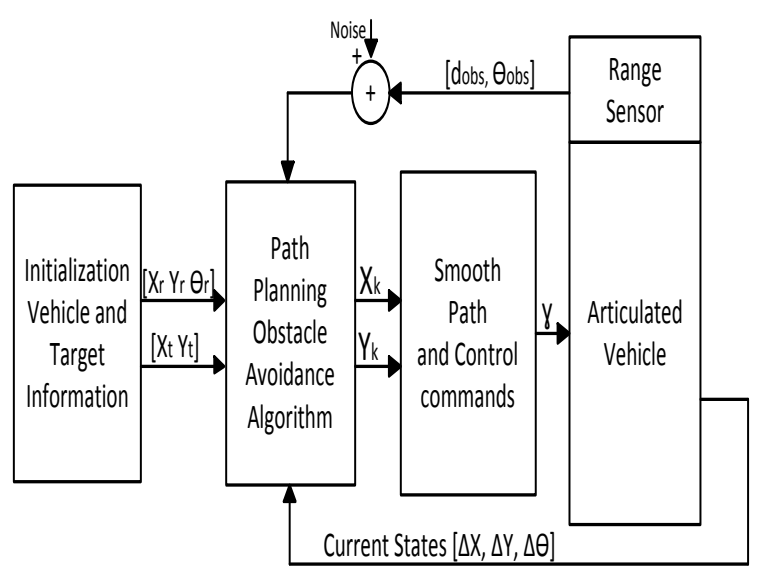

Figure 3: Block diagram of the proposed path planning algorithm based on the nonlinear articulated kinematic model.

for the displacement and orientation of the vehicle.

The assumed sensory system is able to detect the obstacles and the surrounding environment, measure the distance of the articulated robot from the obstacle $d_{o b s} \in \mathfrak{R}$, while a sensing radius $\theta_{\text {obs }} \in \mathfrak{R}$ is being considered, reflecting real life sensing limitations. In the presented approach all the obstacles and the surrounding environment are being considered as point clouds in a 2-dimensional space, while the overlapping obstacles are being clustered and represented by a single and unified obstacle.

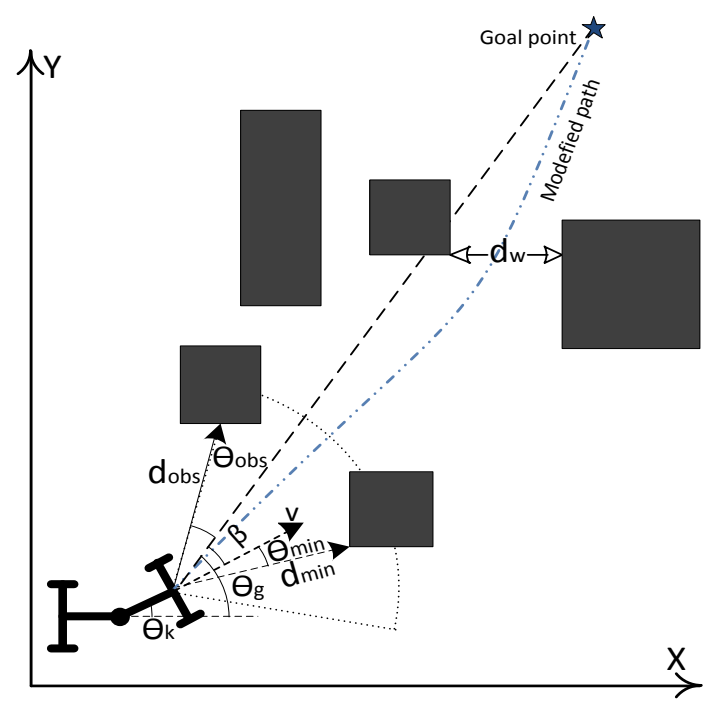

Figure 4: Notations and overall concept of the proposed path planning algorithm.

The overall flowchart diagram for path planning and obstacle avoidance for the case of an articulated vehicle is being depicted in Figure 5, while is can be summarized as it follows:

[Step 1: Initialization] Define initial $\left[X_{r} Y_{r} \theta_{r} \dot{\gamma}_{r}\right]$ and

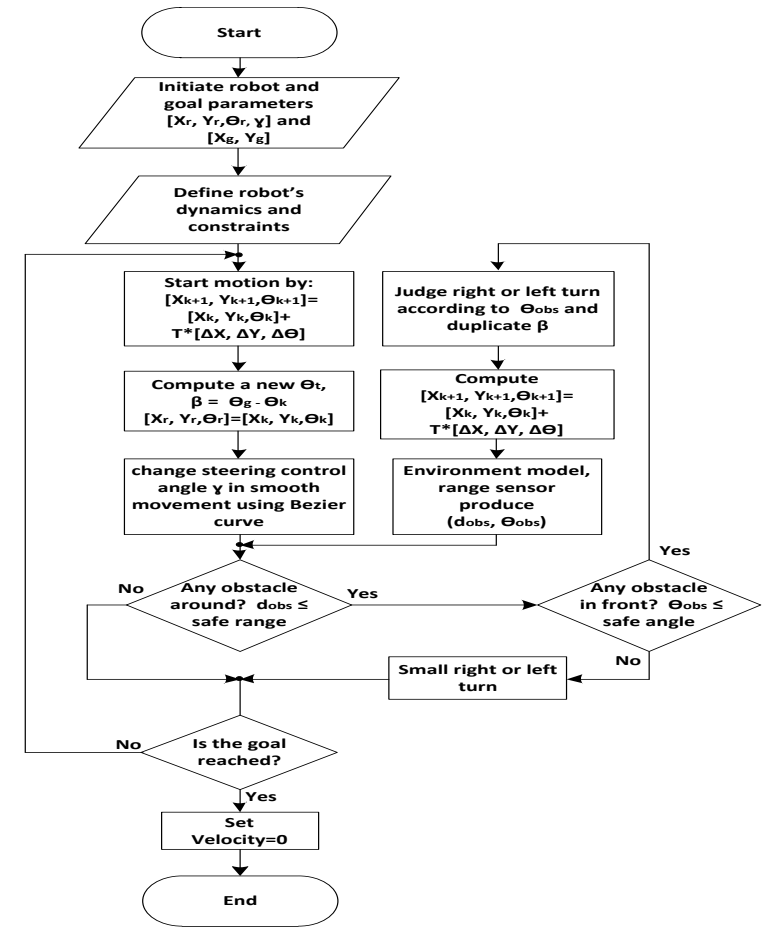

Figure 5: Main flowchart of path planning motion.

goal $\left[X_{g} Y_{g}\right]$, define the articulated vehicle's specific parameters $V, d_{\min }, \theta_{\min }, d_{o b s}, \theta_{o b s}$, the path update rate defined as $T$ and the vehicle's mechanical and physical constraints that needs to be taken under consideration. Set $\left[X_{k} Y_{k} \theta_{k}\right]=\left[\begin{array}{lll}X_{r} & Y_{r} & \theta_{r} \\ \dot{\gamma}_{r}\end{array}\right]$, with $k \in Z^{+}$ the sample index.

[Step 2: Path Update] Utilize Equations (1), (2), (6), (7) and (8) to update the coordinates of the next way point as:

$\left[\begin{array}{llll}X_{k+1} & Y_{k+1} & \theta_{k+1}\end{array}\right]=\left[\begin{array}{lll}X_{k} & Y_{k} & \theta_{k}\end{array}\right]+T *[\Delta X \Delta Y \Delta \theta]$

calculate:

$$
\begin{aligned}
\theta_{g} & =\arctan \frac{Y_{k+1}-Y_{g}}{X_{k+1}-X_{g}} \\
\beta & =\theta_{g}-\theta_{k}
\end{aligned}
$$

to produce $\gamma$ with $\theta_{g}$ the angle between the line that connects the center of gravity of the vehicle's front part to the goal point and the $X$ axis, while $\beta$ is the difference angle among the vehicle's orientation angle, with the $X$ axis and $\theta_{g}$. During the application of this step, constraints can be imposed on the articulated vehicle just by bounding the allowable articulation within $\gamma^{+} \leq \dot{\gamma} \leq \gamma^{-}$, with ${ }^{+}$and ${ }^{-}$representing the maximum and the minimum bounds on the articulated angle.

[Step 3: Obstacle Avoidance] The obstacle avoidance strategy becomes active when the safety conditions $d_{\text {min }}$ and $\theta_{\text {min }}$ are satisfied. This can be evaluated 
by calculating the update of the distance from the obstacle and the obstacle's angle by:

$$
\begin{aligned}
D i_{o b s} & =\sqrt{\left(X_{k+1}-X_{o b s}\right)^{2}+\left(Y_{k+1}-Y_{o b s}\right)^{2}} \\
\theta_{o b s} & =\arctan \frac{Y_{k+1}-Y_{o b s}}{X_{k+1}-X_{o b s}} \\
\theta_{o b s, g} & =\theta_{o b s}-\theta_{g}
\end{aligned}
$$

while in the case that the following conditions are true:

$$
\begin{array}{ccc}
\left(\text { Dis }_{\text {obs }}<d_{\text {min }}\right) & \text { AND } & \left(\theta_{\text {obs }, g}<\theta_{\text {min }}\right) \\
& \text { OR } & \\
\left(\text { Dis }_{\text {obs }}<d_{\text {min }}\right) & \text { AND } & \left(\theta_{\text {obs }, g}<-\theta_{\text {min }}\right)
\end{array}
$$

the changing in the steering angle is being duplicated and Step 3 is repeated again till the condition in (9) is false and the algorithm continues from Step 2 or the bounds on the articulated angle cannot meet and the algorithm jumps to Step 4.

[Step 4: Reaching Final Goal] If $\left[\begin{array}{ll}X_{k+1} & Y_{k+1}\end{array}\right]=$ $\left[\begin{array}{ll}X_{g} & Y_{g}\end{array}\right] \pm$ Dis $_{\text {tolr }}$, set velocity $=0$ and the path planning algorithm has been terminated. Otherwise, the algorithm jumps to Step 2 and the whole process is being repeated in order to avoid collisions with the obstacles until vehicle reaches the goal tolerance distance.

During the execution of the proposed path planning algorithm, and especially Step 2, the proposed path planning algorithm always smoothes the produced way points by the utilization of Bezier curve filtering. The mathematical formulation of the applied Bezier smothering is denoted as:

$$
\mathbf{B}(t)=\sum_{i=0}^{n}\left(\begin{array}{l}
n \\
i
\end{array}\right)(1-t)^{n-i} t^{i} P_{i}, t \in[0,1]
$$

The number $n$ of the considered control points for the Bezier curve generation plays a significant role in the final shape of the produced smooth path as it can be observed from Figure 6, where multiple Bezier lines are being displayed with respect to different number of control points. A $n$ degree Bezier line always passes through the first and last control points and it can be proved that it always lies within the convex hull of the control points, while being tangent to the lines connecting the way points (Chaudhry et al., 2010).

\section{SIMULATION RESULTS}

For simulating the efficacy of the proposed path planner, the following articulated vehicle's characteristics have been considered: $l_{1}=l_{2}=0.6 \mathrm{~m}, \mathrm{~W}=0.58 \mathrm{~m}$, while the vehicle's speed is constant and equal to

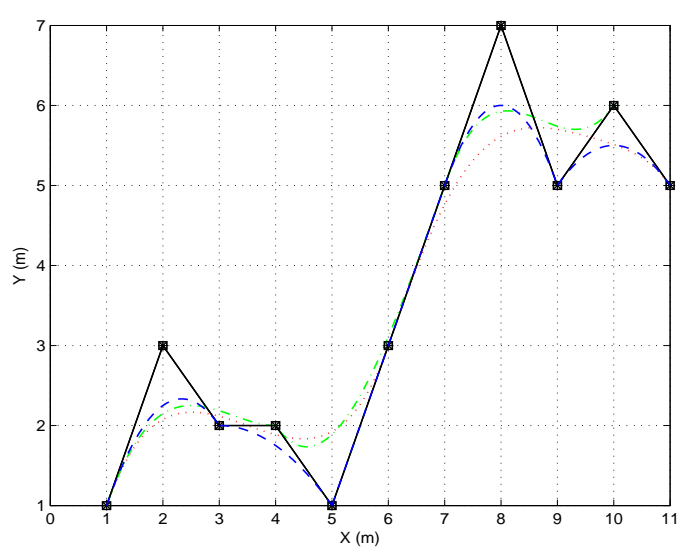

Figure 6: Bezier curves based on different number of control points resulting in different paths, (Black line-solid path produced from the generated way points, red line-dots for 6 points, green line-dash dot for 4 points and the blue linedash for 3 points).

$1 m$ sec. Moreover, the constraints imposed on the articulated angle $\gamma$ have been defined as $\pm 0.523 \mathrm{rad}$ and random measurement Gaussian noise with a fixed variance was added to all measurements of the range sensor to simulate the real life measurement distortion.

The effectiveness of the proposed algorithm will be evaluated in arenas of different types and dimensions. More analytically, the algorithm is simulated on three types of environments with different obstacle configurations, where the vehicle and obstacle geometry is described in a 2D workspace. The obtained outputs of the path planning solutions from the indicated starting points to the goal points is being depicted in Figures 7, 8 and 9, displaying cases with the same sensing radius $d_{o b s}=3 m$ and various $d_{\text {min }}$.

As it can be observed in all the examined cases the vehicle is able to avoid all the obstacles, including the bounding surrounding (e.g. walls), which can also be considered as obstacles without loss of generality. In the presented simulations, the articulated vehicle is achieving to reach the reference final goal, independently of the initial vehicle's orientation, while in all the simulations the safety radius has been also displayed with the red circle notation. The basic assumption in all these simulations is that the articulated vehicle, in every time instant, is aware of the coordinates of the final goal and thus the path is being tuned in every step based on the identified obstacles, while the vehicle explores the surrounding environment towards the final goal. In an obstacle free environment, the optimal solution to this problem would have been a straight line connecting the initial with the goal point, a case that can be easily identified in the presented simulation in Figure 7. The online iden- 


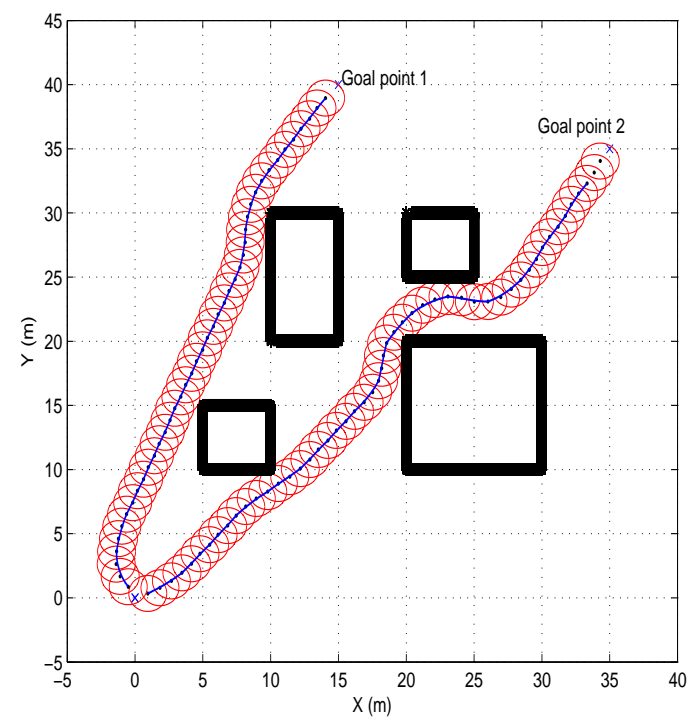

Figure 7: Different shape obstacles placed in the workspace. During these simulations the vehicle starts from different initial angles at $\left[0,0,120^{0}, 7.5^{0}\right]$ and $\left[0,0,20^{0}, 7.5^{\circ}\right]$ to reach the goal points located at $[15,40]$ and $[35,35]$ respectively, with safe distance $d_{\min }=3 m, d_{o b s}=3 m$.

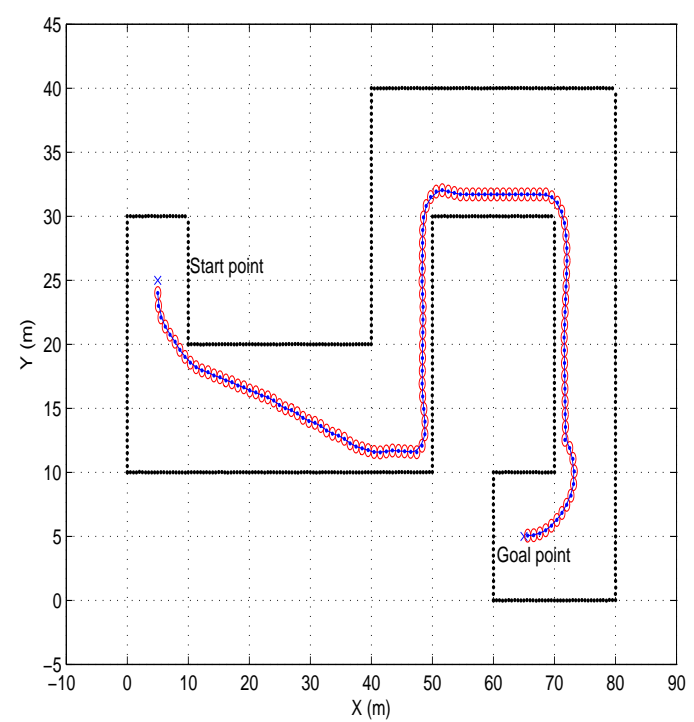

Figure 8: Path planning in an arena having boundaries on both sides of the road a fact that restricts the articulated vehicle motions. During this simulation scenario the vehicle is starting from the initial posture $\left[5,25,-90^{\circ}, 7.5^{\circ}\right]$ and the goal is located at $[65,5]$ and $d_{\min }=0.5 \mathrm{~m}, d_{o b s}=3 \mathrm{~m}$.

tification of obstacles produces distortions from following the straight line, connecting the robot with the final goal point, while the sensing and safety radius are having a major effect on the path calculation. As it can observed in Figure 10, the safety radius plays a very significant role in shape of the path. In this Fig-

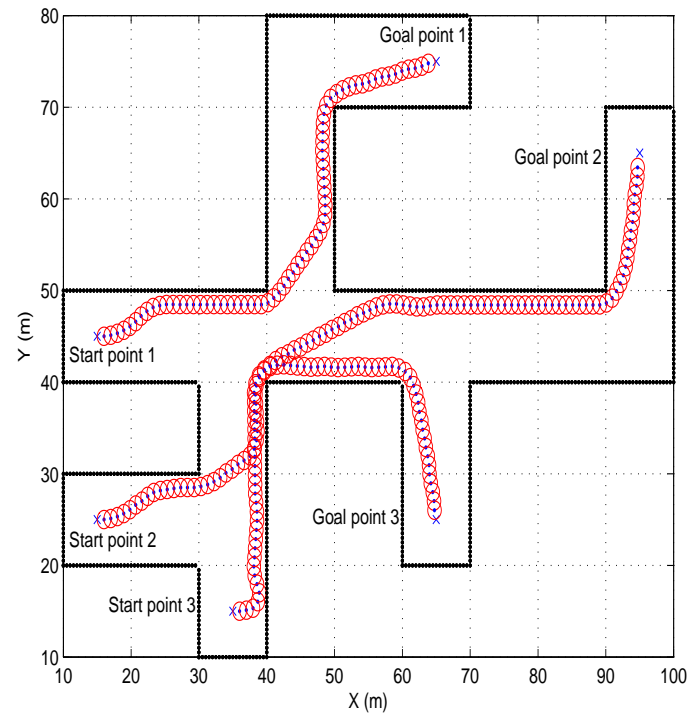

Figure 9: Path planning in an arena having more complicated boundaries on both sides of the road, with noise measurement. The scenario is starting from different initial postures $[15,45],[15,25]$ and $[35,15]$ with initial $[\theta, \gamma]=$ $\left[10^{\circ}, 7.5^{\circ}\right]$ and the goals are located at $[65,75],[95,65]$ and $[65,25]$ respectively with $d_{\min }=1.0 m, d_{o b s}=3 m$.

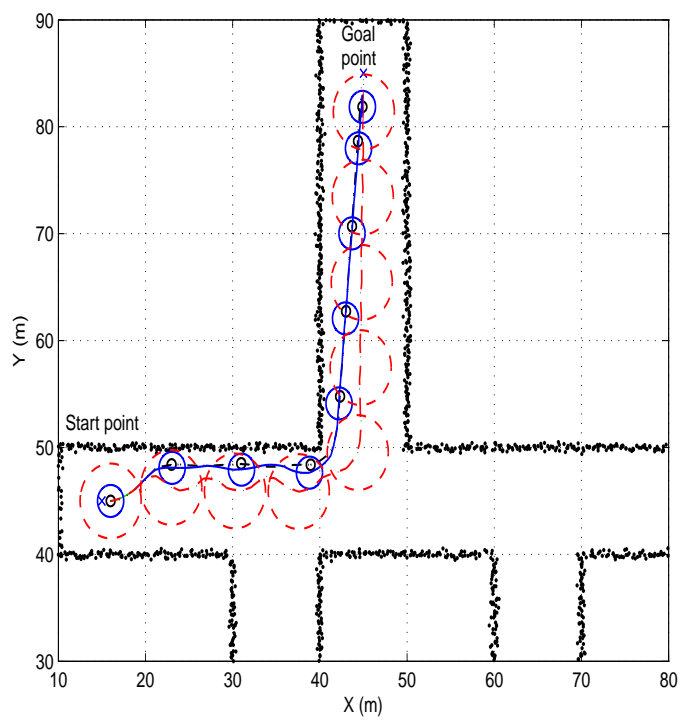

Figure 10: During these simulations the vehicle is starting from the initial posture $\left[15,45,0,5^{0}\right]$ and the goal point is located at $[45,85]$, while during movement different safe distances $d_{\text {min }}$ have been utilized as $0.5,1.5$ and $3.5 \mathrm{~m}$, with the same $d_{o b s}=3 m$.

ure, different paths with different safe distances $d_{\text {min }}$ and with the same sensing radius $d_{o b s}$ are being presented. In the case that the vehicle is moving in a bounded space, the selection of a relatively big safety radius introduces oscillations in the translation of the robot due to sequential safety violations that produce 
corresponding change in the direction of the vehicle for avoiding the obstacle. In case that small safety radius are being selected, this effect is being vanished and smooth and shorter, non oscillatory, paths can be produced.

Finally it should be stated that the arenas in Figures 8 and 9 are typical realistic examples of areas where articulated vehicles operate, as the mine tunnels and the civil roads are. In the presented simulations the consideration of the articulated vehicle's dynamic motion is obvious especially in the time instances where the vehicle is turning towards the goal and while performing at the same time obstacle avoidance. This effect is of paramount importance for the case of articulated vehicles as classical point dynamic approaches in path planning will obviously results in non-realistically achievable paths that would directly lead to collisions.

\section{CONCLUSIONS}

In this article a novel online dynamic smooth path planning scheme based on a bug like modified path planning algorithm for an articulated vehicle under limited and sensory reconstructed surrounding static environment has been proposed. In the presented approach factors such as the real dynamics of the articulated vehicle, the initial and the goal configuration, the minimum and total travel distance between the current and the goal points, the geometry of the operational space, and the path smothering approach based on Bezier lines have been taken under consideration to produce a proper path for an articulated vehicle, which can be followed by correspondingly altering the vehicle's articulated angle. The efficiency of the proposed scheme has been evaluated by multiple simulation studies.

\section{REFERENCES}

Buniyamin, N., Wan Ngah, W., Sariff, N., and Mohamad, Z. (2011). A simple local path planning algorithm for autonomous mobile robots. International journal of systems applications, Engineering \& development, 5(2):151-159.

Chaudhry, T., Gulrez, T., Zia, A., and Zaheer, S. (2010). Bézier curve based dynamic obstacle avoidance and trajectory learning for autonomous mobile robots. In Intelligent Systems Design and Applications (ISDA), 2010 10th International Conference on, pages 10591065. IEEE.

Chazelle, B. (1987). Approximation and decomposition of shapes. Advances in Robotics, 1:145-185.
Ge, S. and Cui, Y. (2000). New potential functions for mobile robot path planning. Robotics and Automation, IEEE Transactions on, 16(5):615-620.

Guechi, E., Lauber, J., and Dambrine, M. (2008). On-line moving-obstacle avoidance using piecewise bezier curves with unknown obstacle trajectory. In Control and Automation, 2008 16th Mediterranean Conference on, pages 505-510. IEEE.

Kamon, I., Rimon, E., and Rivlin, E. (1998). Tangentbug: A range-sensor-based navigation algorithm. The International Journal of Robotics Research, 17(9):934953.

Kamon, I. and Rivlin, E. (1997). Sensory-based motion planning with global proofs. Robotics and Automation, IEEE Transactions on, 13(6):814-822.

Lumelsky, V. and Skewis, T. (1990). Incorporating range sensing in the robot navigation function. Systems, Man and Cybernetics, IEEE Transactions on, 20(5):1058-1069.

Lumelsky, V. and Stepanov, A. (1986). Dynamic path planning for a mobile automaton with limited information on the environment. Automatic Control, IEEE Transactions on, 31(11):1058-1063.

Nayl, T., Nikolakopoulos, G., and Guastafsson, T. (2011). Kinematic modeling and simulation studies of a lhd vehicle under slip angles. In Computational Intelligence and Bioinformatics/755: Modelling, Identification, and Simulation. ACTA Press.

$\mathrm{Ng}$, J. and Bräunl, T. (2007). Performance comparison of bug navigation algorithms. Journal of Intelligent \& Robotic Systems, 50(1):73-84.

Nilsson, N. (1969). A mobile automaton: An application of artificial intelligence techniques. Technical report, DTIC Document.

Roberts, J., Duff, E., Corke, P., Sikka, P., Winstanley, G., and Cunningham, J. (2000). Autonomous control of underground mining vehicles using reactive navigation. In Robotics and Automation, 2000. Proceedings. ICRA'O0. IEEE International Conference on, volume 4, pages 3790-3795. IEEE.

Scheding, S., Dissanayake, G., Nebot, E., and DurrantWhyte, H. (1999). An experiment in autonomous navigation of an underground mining vehicle. Robotics and Automation, IEEE Transactions on, 15(1):85-95.

Škrjanc, I. and Klančar, G. (2010). Optimal cooperative collision avoidance between multiple robots based on bernstein-bézier curves. Robotics and Autonomous systems, 58(1):1-9.

Usher, K. (2006). Obstacle avoidance for a non-holonomic vehicle using occupancy grids. In 2006 Australasian Conference on Robotics and Automation. 